Federal Reserve Bank of Minneapolis

Research Department Staff Report 282

November 2000

\title{
On the Equilibrium Concept for Overlapping Generations Organizations
}

\author{
Edward C. Prescott* \\ Federal Reserve Bank of Minneapolis \\ and University of Minnesota \\ José-Víctor Ríos-Rull* \\ University of Pennsylvania, \\ Centre for Economic Policy Research, \\ and National Bureau of Economic Research
}

\begin{abstract}
A necessary feature for equilibrium is that beliefs about the behavior of other agents are rational. We argue that in stationary OLG environments this implies that any future generation in the same situation as the initial generation must do as well as the initial generation did in that situation. We conclude that the existing equilibrium concepts in the literature do not satisfy this condition. We then propose an alternative equilibrium concept, organizational equilibrium, that satisfies this condition. We show that equilibrium exists, it is unique, and it improves over autarky without achieving optimality. Moreover, the equilibrium can be readily found by solving a maximization program.
\end{abstract}

*Ríos-Rull thanks the National Science Foundation for Grant SBR-9309514 and the University of Pennsylvania Research Foundation for their support. Prescott thanks the National Science Foundation for grant SBR-9996201. We thank Julio Davila, Igor Livshits, Larry Jones, Dan Silverman, and attendants at seminars in the Penn macro lunch group, Federal Reserve Bank of Minneapolis, Cornell-Penn State University Macro-Theory Conference, the University of Rochester, UCLA, and the 1999 Midwest Theory Meetings for comments. The views expressed herein are those of the authors and not necessarily those of the Federal Reserve Bank of Minneapolis or the Federal Reserve System. 


\section{Introduction}

In this paper we propose a new equilibrium concept for stationary OLG environments, organizational equilibrium. The equilibrium allocation exists and is essentially unique, as it is the solution to a well-behaved maximization problem. The equilibrium allocation is much better than autarky but is not Pareto optimal, and it is very different from that implied by the standard equilibrium concepts.

A necessary feature of an equilibrium concept is that beliefs about the behavior of other agents are rational. In stationary OLG environments agents of any future generation have the option of restarting, perhaps by moving along with the seeds of future generations to another island. These two features imply that any future generation must do as well as the initial generation did. Existing equilibrium concepts in the literature do not satisfy this condition.

The organizational equilibrium concept is applicable to other environments where there is a role for contractual arrangements that outlive their founders, which from now on we refer to as organizations. Examples of organizations are firms, criminal gangs, economics departments, guilds and governments, to cite only a familiar few. The key elements of organizations are that in the future new members join and, when old, run the organization and that the initial generation of old agents cannot dictate the behavior of future generations. With finite horizon problems, the planning problem can be solved by backward induction. With infinite horizon problems, for the reasons pointed out by Shell (1971) and for other reasons, the analysis becomes much more interesting.

In section 2 we discuss the environment: preferences and endowment. We place special emphasis on the fact that agents of all generations have the same opportunities. Even though we proceed with the analysis in the context of a stationary example, we later present our definition 
of equilibrium under certain forms of non-stationarity. Section 3 formally describes the participation, or no restarting, constraints that rationality imposes and that we think equilibrium should satisfy. In section 4 we review the standard notions of equilibrium—core, market and game theoretical - that apply to the overlapping generations environment and show how they either fail to exist, violate rationality, or yield multiplicity of equilibria to the point that just about any allocation is an equilibrium outcome. In section 5 we describe our favorite allocation and some of its properties. Section 6 formally defines our equilibrium concept that generates our allocation and that we propose as the appropriate equilibrium concept for this class of environments. Section 7 discusses how to incorporate assets into our equilibrium concept and shows how the implied equilibrium relates to the competitive one. In Section 8 we discuss the extension of our equilibrium concept to non-stationary environments, and we provide an example for a growing economy. Further, we discuss the extension of this concept to other types of environments. Section 9 concludes.

\section{The Environment}

The environment is an old favorite in economics: the stationary, two period-lived, one good per period, no production, overlapping generations economy. We will deal with a precise example of this economy in the first few sections because the arguments are easier to follow. Later we will use a more general specification.

In each period $t=\{0,1,2, \ldots\}$, a new agent is born ${ }^{1}$ who goes on to live two consecutive periods. The endowment of the agents is stationary and equal to $\{3,1\}$. Generation $t$ agents' preferences are represented by the utility function $u_{t}=u\left(y_{t}, z_{t+1}\right)=\log \left(y_{t}\right)+\log \left(z_{t+1}\right)$. Note that we use the letter $y$ to refer to young and the letter $z$ to refer to old and the subscript to refer to the period; utilities are indexed by the date of birth. A very important feature of this environment is that at 
any point in time the young agents can leave the economy and start another one. If they do so, the next generation will be born in the new location. We say that they move to another island, although literally, all that this means is that they ostracize the old and become, de facto, the first old themselves.

Denote with $a_{t} \in A=[0,3]$ the possible transfers that agent $t>0$ can give to his elders. For compactness of notation we sometimes write $u_{t}=U_{t}(a)=U\left(a_{t}, a_{t+1}\right)=\log \left(3-a_{t}\right)+\log \left(1+a_{t+1}\right)$. In this environment note that any constant transfer in the interval $[0,1]$ improves the welfare of everybody. Moreover, this improvement is monotonic in the transfer within this interval. Of course, many other transfer schemes that are non-stationary are also possible. The allocation $a_{t}=$ 1 for all $t$ is particularly interesting, and we call it the optimal stationary allocation. It is both stationary and Pareto optimal. It implies the maximum sustainable utility for all agents. This allocation is depicted as point A in Figure 1, while point B is the endowment, or autarky.

We turn next to discuss the implications of agents having the restarting option.

\section{Implications of the Possibility of Agents Leaving}

In this environment any agent can choose to move to another island and hence become the first old. Rationality of individual behavior requires that the utility obtained by the first old cannot be higher than that of any agent who comes later: if this were the case, the latter would move to another island and achieve as the first old the utility of the former. ${ }^{2}$ This introduces certain participation constraints that we call the "no restarting condition." Formally, let $\left\{a_{t}\right\}_{t=1}^{\infty}$ be a set of transfers. Then we have

Definition 1 A set of transfers $\left\{a_{t}\right\}_{t=1}^{\infty}$ satisfies the no restarting condition if

$$
U\left(a_{t}, a_{t+1}\right) \geq U\left(0, a_{0}\right) .
$$


Or alternatively, $U_{t}(a) \geq U_{0}(a)$, or $\left\{y_{t}, z_{t}\right\}_{t=0}^{\infty}$ satisfies $u\left(y_{t}, z_{t+1}\right) \geq u\left(3, z_{0}\right)$.

In particular, the optimal stationary allocation cannot be implemented through an equilibrium concept that satisfies this Condition. The reason is clear: the first old manages to achieve a utility level that is much higher (as he consumes $\{3,2\}$ ) than younger generations who only get the utility of $\{2,2\}$. If such an allocation were achievable, younger generations would be better off by moving to another island and restarting the economy. This fact will be a recurrent theme of this paper.

\section{How do Equilibrium Concepts Fare?}

We now review a number of equilibrium concepts that can be applied to this class of economies in order to pick an allocation. We show how they predict allocations that do not satisfy the no restarting condition. As we will see below this is not exactly true: non-cooperative game theoretical notions have a large set of equilibria, some of which are rational in the sense described and some not. Also, the core is empty. We classify these concepts into core, market and game theoretical equilibrium concepts.

\subsection{The Core}

Esteban (1986) and Hendricks, Judd, and Kovenock (1980) show that economies of the type that we are considering have an empty core because coalitions of later agents can always do better by themselves than by giving anything to the previous agents. Engineer, Esteban, and Sakovics (1997) propose a notion of institutions that are needed to implement a transfer and are expensive to change and find that under certain conditions it is in the interest of the first old to build them. Unfortunately, institutions are not modeled explicitly, and we found this to be a shortcoming of their analysis. 
We see the standard definition of the core as being too restrictive: blocking coalitions can always be constructed under the traditional definition of the core. This requisite prevents any transfer. As we will see later, our concept of organizational equilibrium requires an organization that cannot be blocked by another organization by means of a certain restrictive type of allocations or consumption plans. In this sense, our equilibrium concept can be thought of as a form of recursive core. $^{3}$

\subsection{Market Notions of Equilibrium}

Even though this is a model designed to study monetary economics, ${ }^{4}$ non-monetary competitive equilibria can also be readily defined. We review the concepts of equilibrium with and without money.

\subsubsection{Non-Monetary Equilibria}

In this environment, a Debreu (1954) valuation equilibrium can be readily defined. Let the commodity space be

$$
S=\left\{s \in \Re^{\infty}|\sup | 4^{t} s_{t} \mid<\infty\right\}
$$

with norm $\|s\|_{s}=\sup _{t}\left|4^{t} s_{t}\right|$. Note that this commodity space is essentially an $l^{\infty}$ space with the individual components rescaled by the factor $4^{t}$. Note that individual endowments belong to the space, while the aggregate endowment does not. A consequence of this latter fact is that the First Welfare Theorem fails. Also note that any individual allocation also belongs to the space.

A valuation equilibrium for this environment consists of the price $p^{*}=\left\{p_{t}^{*}\right\}_{t=0}^{\infty}=\left\{3^{t}\right\}_{t=0}^{\infty}$ and the autarkic allocation. ${ }^{5}$ First note that $p^{*}$ is a bona fide price in the sense that it defines a continuous linear functional on the commodity space. ${ }^{6}$ Second note the price is chosen so that 
relative prices equate the marginal rate of substitution at autarky. Finally, feasibility is immediate.

As we noted above, this equilibrium is not optimal. Far from it, there are a large number of transfer schemes from the young to the old, all of which are Pareto improving. ${ }^{7}$

The equilibrium is unique up to the numeraire. One way to prove this result is as follows. A difference equation specifying $\left(y_{t+1}, p_{t+1}\right)$ as a function of $\left(y_{t}, p_{t}\right)$ is obtained by solving the budget constraint and first order condition of generation $t$ along with the date $t+1$ market clearing condition. The initial value is $\left(y_{0}=\xi, p_{0}=1\right)$.

The same conclusions follow for "sequence-of-market" equilibria. In such a setup, only trades within generations are feasible, and given common convex preferences there are no mutually beneficial trades, rendering autarky as the only equilibrium allocation. The relative prices of the date $t$ and date $t+1$ goods are the same as for the valuation equilibrium.

To summarize, a non-monetary equilibrium exists both as a valuation equilibrium and as a sequence of market equilibrium. It is unique, and it yields abysmal utilities to all agents since the equilibrium is autarky.

\subsubsection{Monetary Equilibria}

This is the basic model where monetary equilibria has been studied. Its main properties are superbly described in Wallace (1980). In a monetary equilibrium, the problem faced by agent $t$ can be written as

$$
\begin{gathered}
\max _{y_{t}, m_{t}, z_{t+1}}\left\{\log y_{t}+\log z_{t+1}\right\} \quad \text { subject to } \\
y_{t}+m_{t} q_{t}=3 \\
z_{t+1}=1+m_{t} q_{t+1},
\end{gathered}
$$


where $m_{t}$ is the nominal holdings of money chosen by the agent and $q_{t}$ is the date $t$ price of money (the inverse of the price level). In equilibrium, total money acquired has to equal total money in the economy that we normalize, so $m_{t}=1$ for all $t$. Substituting the equilibrium quantity of money in the first order condition of the households yields

$$
q_{t+1}=q_{t} /\left(3-2 q_{t}\right)
$$

This condition has to be satisfied by the equilibrium prices. Note that if $q_{0}>1$, successive application of (6) shows that eventually the price will be negative, which is inconsistent with equilibrium. Note also that if $q_{0}<1$ then $\lim _{t \rightarrow \infty} q_{t}=0$, and the allocation converges to autarky. If $q_{0}=$ 1 then the allocation is the stationary optimal one. This reasoning shows that for any $q_{0} \in(0,1]$, there is a monetary equilibrium indexed by $q_{0}$.

In words, there is a continuum of equilibria. One yields the optimal stationary allocation that, as stated above, does not satisfy the no restarting condition. The other equilibria give utility lower than that to the first agent, but sufficiently far into the future there are agents with utility arbitrarily close to that in autarky, which again violates the no restarting condition.

The no restarting condition can be more graphically described in the context of monetary equilibria. Suppose some old agent tries to sell his money to the young. Suppose also that the old agent's allocation in the monetary equilibria yields higher utility than that of the young agent. Then, the no restarting condition states that the young agent should say,

"Thanks for the idea. I think I might as well implement money myself and be the first old."

Note that this is feasible given that money is some intrinsically useless and costless commodity. To summarize, monetary equilibria can yield the optimal allocation and some other allocations 
that Pareto dominate autarky. However, none of these allocations satisfy the no restarting conditions, which violates rationality.

Another conceptual problem with non-monetary competitive equilibria for this environment is the lack of equivalence between the core and the set of competitive equilibria in large economies. Autarky is a non-monetary competitive equilibrium while the core is empty.

\subsection{Game Theoretical Notions of Equilibria}

To use game theoretical notions of equilibria we need a concept of a game. Unfortunately the environment that we are interested in, unlike many others, does not define by itself a particular game to be played. Hammond (1975) defined a simple transfer game that may represent some of the key properties of the OLG environment, and that is the one typically associated with the environment. In this game, the set of actions for each agent $t, A_{t}$, consists of the transfers he can give to his elder. The elder in turn does nothing. He accepts the transfer and dies.

Define a period- $t$ history as $h_{t}=\left\{0, a_{1}, a_{2}, \ldots, a_{t-1}\right\}$ and the set of all possible period- $t$ histories as $H_{t}=A_{1} \times A_{2} \times \ldots \times A_{t-1}$. A strategy for agent $t$ is a mapping $s_{t}: H_{t} \rightarrow A_{t}$. A strategy profile for the game is $s=\left\{s_{t}\right\}_{t=0}^{\infty}$. Define $S_{t}$ as the set of all possible strategies for agent $t$ and $S=S_{1} \times S_{2} \times \ldots$

Note that in this game the option of moving to another island is not clearly defined. We could perhaps think of a zero transfer as moving to another island, but such an action could be also thought of differently.

We now review the main equilibrium concepts for dynamic games. We consider subgame perfection a requirement for rationality in complete information games and therefore a requirement for equilibrium. 


\subsubsection{Subgame Perfection}

Definition 2 A subgame perfect equilibrium is a strategy profile $\hat{s}$ such that

$$
\hat{s}_{t} \in \arg \max _{a \in A} U_{t}\left(a, \hat{s}_{t+1}\left(h_{t}, a\right)\right) \quad \text { for all } \mathrm{t} \text {, for all } h_{t} .
$$

Hammond (1975) already noted that the set of all subgame perfect equilibrium includes strategy profiles supporting an autarkic outcome. An example is

$$
\hat{s}_{t}\left(h_{t}\right)=0 \quad \text { for all } t \text {, for all } h_{t} \text {. }
$$

He also noted the set of subgame perfect equilibria includes as well strategy profiles that support the stationary Pareto optimal outcome. An example is

$$
\hat{s}_{t}\left(h_{t}\right)=1 \text { if } h_{t}=\{1,1, \cdots, 1\} \text {, and } \hat{s}_{t}\left(h_{t}\right)=0 \text { otherwise. }
$$

Moreover, the allocation $a_{t}=2$ for all $t$ can also be implemented as a subgame perfect equilibrium with the strategy,

$$
\hat{s}_{t}\left(h_{t}\right)=2 \text { if } h_{t}=\{2,2, \ldots, 2\} \text {, and } \hat{s}_{t}\left(h_{t}\right)=0 \text { otherwise }
$$

This allocation is interesting. It makes all but the first generation indifferent with autarky, but it makes the first generation extremely happy, as its consumption allocation is the point $\{3,3\}$. It is in fact a Pareto optimal allocation.

Needless to say this allocation does not satisfy the no restarting condition, as any agent who restarts the system would obtain $U(0,2)$, rather than $U(2,2)$.

\section{Equilibria preferred by the old}

What if the first old chose strategies subject to those strategies being subgame perfect as used, for example, in Boldrin and Rustichini (2000)? However, as we have seen, the solutions to this problem, strategies that yield $a_{0}=2$, do not satisfy the no restarting condition. 


\subsubsection{Asheim's Revision Proofness}

Asheim (1997) proposes a refinement of subgame perfection that imposes some sensible restrictions in the possible outcomes. However, Silverman (1999) has shown that the set of allocations that are implementable through revision proof strategies is still quite large, and, in particular, it contains the stationary Pareto optimal allocation associated to a constant transfer of 1. We have already seen that this allocation does not satisfy the no restarting condition.

\section{Equilibrium preferred by the old}

Equilibria preferred by the old among those that are revision proof yield $a_{0} \geq 1$, which does not satisfy the no restarting condition.

\subsubsection{Kocherlakota's Reconsideration Proofness}

Kocherlakota (1996) considers the problem facing an infinitely lived decision-maker with time inconsistent preferences. The environments that he considers are stationary. He represents the problem as a dynamic game played by the agent's different selves. He introduces an equilibrium refinement for infinite horizon, complete information, stationary games with a single decision-maker in each period. Here stationary means that all subgames are the same. A subgame perfect equilibrium is symmetric if after every history, the continuation path has the same value. A symmetric subgame perfect equilibrium is reconsideration proof if it yields the highest value in the symmetric set of equilibria.

The obvious extension of his refinement concept to the Hammond (1975) transfer game yields our preferred allocation. We do not know what would happen for all possible games that we could associate to this environment. Neither do we know how to extend this concept to the environments with assets or with growing endowments that are addressed in this paper. 


\section{The Proposed Allocation}

So far we have shown that none of the standard equilibrium concepts leads us to an allocation that satisfies our no restarting condition and improves upon autarky. We now turn to the question of whether agents can improve upon autarky while not violating the no restarting condition.

There are many allocations that improve upon autarky. We think the following allocation should be the outcome for any suitable equilibrium concept for our class of environments.

The allocation gives all generations utility level $2 \log 2$, which is the maximal sustainable utility. The transfers by generation 1 solve

$$
\log 3+\log \left(1+\hat{a}_{1}\right)=2 \log 2 \Rightarrow \hat{a}_{1}=1 / 3 .
$$

For our proposed allocation to be individually rational, an allocation must yield at least $2 \log 2$ to those born in 1 . This means that $\hat{a}_{2}$ has to solve

$$
\log \left(3-\hat{a}_{1}\right)+\log \left(1+\hat{a}_{2}\right)=2 \log 2 \Rightarrow \hat{a}_{2}=1 / 2 \text {. }
$$

In general $\hat{a}_{t}$ solves

$$
\log \left(3-\hat{a}_{t-1}\right)+\log \left(1+\hat{a}_{t}\right)=2 \log 2
$$

yielding $\hat{a}_{t}=t /(t+2)$. Note that

$$
\lim _{t \rightarrow \infty} \hat{a}_{t}=1 .
$$

A few things to note about this allocation are depicted in Figure 2.

1. It is resource feasible.

2. No generation has the incentive to restart the system if this is the equilibrium allocation. 
3. It is not a market equilibrium, either monetary or non-monetary (recall that the former is either the stationary Pareto optimal allocation or an allocation that converges to autarky, while only autarky is a non-monetary equilibrium).

4. Even though this allocation can be implemented through a subgame perfect Nash equilibrium, or even a revision proof equilibrium, it is one of many that has this property. Moreover, it would not be singled out by the first old if he were to choose among those equilibria in either game theoretical class.

So far this seems like a nice insight; we want more. We want a procedure to find allocations like this one. We turn to this issue next.

\section{Organizational Equilibrium}

To define the equilibrium concept, we look at a general class of stationary economies with generic young and old endowments $\left\{e^{y}, e^{z}\right\}$ and a generic concave, increasing, continuous utility function. Again let the utility be denoted by $u$. Let the set of feasible allocations be denoted by

$$
F=\left\{(y, z) \mid y_{t}, z_{t} \geq 0, y_{t}+z_{t}=e^{y}+e^{z} \forall t>0 \text { and } y_{0}=e^{y}\right\}
$$

Before explicitly providing our definition of equilibrium, let's look at a program that the first old would solve in order to achieve the maximum possible utility, provided later generations do as well as they do. It is

$$
\max _{v, y, z} u\left(y_{0}, z_{1}\right)
$$

subject to the feasibility constraints

$$
y_{t}+z_{t}=e^{y}+e^{z}, t \geq 1 \text { and } y_{0}=e^{y}
$$

and to the no restarting condition 


$$
u\left(y_{t}, z_{t+1}\right) \geq v, t \geq 1 \text { and } u\left(y_{0}, z_{1}\right) \leq v
$$

Proposition 1 A solution to the program is the set of transfers with the properties that all generations realize the maximal sustainable utility, which is the maximum of $u(y, z)$ subject to $y+z \leq$ $e^{y}+e^{z}$

Proof. The proof is immediate, as the allocation is feasible and no plan exists that yields higher utility to all generations by definition of sustainable.

A strengthening of the no restarting condition yields uniqueness. The strengthening requires that any generation not only can restart the system of transfers, but also can move it backwards. This means that generation $t>s$ can achieve not only the transfer that generation 0 achieves, but also the transfer achieved by generation $s$. A sufficient condition for this stronger version is that agents can only observe the transfer made by the previous generation.

Proposition 2 The solution specified in Proposition 1 satisfies the stronger no restarting condition and is the only solution that satisfies the strong no restarting condition.

Proof. First, the solution does indeed satisfy the stronger version of the no restarting condition, as no generation benefits by moving the system backwards. To establish that it is the only solution, suppose there were another set of transfers that solve program (17)-(18) and that satisfy the strong no restarting condition. The utility of generation zero is determined by the value of the program and therefore the transfer by generation one for all solutions to the program. The strong no restarting condition implies that the utility level of generation $t+1$ weakly exceeds that of generation $t$. If any generation gets higher utility than the initial, then all generations subsequent to that generation must get higher utility than the initial generation. This is impossible, as the initial generation gets the maximum sustainable utility. This establishes the proposition. 
Now we can think of an organization of agents as composed of agents who propose a plan that satisfies the participation and feasibility constraints. Formally,

Definition 3 An organization equilibrium for this environment is a plan $\{\hat{y}, \hat{z}\}$, and associated utilities $\hat{u}_{t}=u\left(\hat{y}_{t}, \hat{z}_{t+1}\right)$, proposed by the original founders such that:

1. The allocation is resource feasible: $\{\hat{y}, \hat{z}\} \in F$.

2. The allocation satisfies the no restarting condition: $\hat{u}_{t} \geq \hat{u}_{0}$ for all $t$.

3. There is no other plan that is both resource feasible and satisfies the no restarting condition for all generations and that yields higher utility for the organization founders.

It is immediate to see that the solution to program (16) satisfies all the conditions of the organizational equilibrium. Moreover, any organizational equilibrium solves that program. The fact that a solution to the program always exists and is unique yields existence and uniqueness of the organizational equilibrium. Before we explore a variation of the economy when there are certain types of assets we note the following.

For this environment, recursive methods do not yield the organizational equilibrium. To see this, consider the following functional equation $T$ :

$$
\begin{aligned}
& v_{m+1}=T\left(v_{m}\right)=\max _{\{y, z\} \in F} u\left(y_{0}, z_{1}\right) \quad \text { subject to } \\
& u\left(y_{t}, z_{t+1}\right) \geq v_{m}, \quad t \geq 1 .
\end{aligned}
$$

If operator $T$ had a maximal fixed point, it would be the organizational equilibrium. ${ }^{8}$ However, $T$ does not have a fixed point. For all $v \leq 2 \log 2, T(v)>2 \log 2$. For $v>2 \log 2$, the constraint set is empty and $T(v)$ is not defined. 


\section{Physical Assets}

We now apply our equilibrium concept to an economy where there are assets that yield a dividend each period and members of the initial generation each own an asset, for example, a tree. Otherwise the economy is identical to the one considered previously. The endowments are $e^{y}=3$ and $e^{z}=1$, and

$$
u\left(x_{t}, z_{t+1}\right)=\log x_{t}+\log z_{t+1} .
$$

The tree bears $\delta>0$ units of goods every period unless it has been destroyed by a previous or the current owner. As we will see later this last assumption of whether the tree can be destroyed by a previous or current owner turns out to be crucially important.

We start analyzing this economy by looking at its competitive equilibrium that turns out to exist and to be unique. Moreover, good prices go to zero exponentially fast. Consequently, all feasible aggregate consumptions have finite values and the First Welfare Theorem applies.

We can write the problem of the agent in the following fashion:

$$
\begin{aligned}
& \max _{y_{t}, m_{t}, z_{t+1}} \log y_{t}+\log z_{t+1} \quad \text { subject to } \\
& y_{t}+m_{t} q_{t}=3 \\
& z_{t+1}=1+m_{t}\left(q_{t+1}+\delta\right),
\end{aligned}
$$

where $q_{t}$ is the tree price in $t$ and $m_{t}$ is the number of shares of the tree that the agent purchases. A competitive equilibrium price system, $\left\{q_{t}\right\}$, has the property that when agents face it, they choose to hold exactly one tree per period, $m_{t}=1$. The first-order conditions for maximization along with market clearing can be used to find the unique competitive equilibrium prices and allocation: 


$$
\begin{aligned}
& q_{t}=\frac{1-\delta+\sqrt{(1-\delta)^{2}+6 \delta}}{2} \\
& y_{t}=\frac{5+\delta-\sqrt{(1-\delta)^{2}+6 \delta}}{2} \\
& z_{t}=\frac{3-\delta+\sqrt{(1-\delta)^{2}+6 \delta}}{2}
\end{aligned}
$$

for all $t \geq 1$ and $y_{0}=3+\delta$.

Before checking whether the competitive equilibrium satisfies the no restarting condition, let's calculate what the organizational equilibrium predicts:

$$
\max _{\{y, z\} \in F} u\left(y_{0}, z_{1}\right)
$$

subject to the no restarting condition, which in this case amounts to

$$
\begin{aligned}
& u\left(y_{t}, z_{t+1}\right) \geq 2 \log 2, \quad t \geq 1 \\
& z_{t} \geq z_{1} .
\end{aligned}
$$

The reason for (29) is that the young can at any time go off to another island on their own, which will yield them utility $2 \log 2$. The reason for (30) is that future old people are in the same situation as first old people. Therefore, they must receive as much for the tree as the first old. The solution to the programming problem is then

$$
z_{t}=\frac{4+\delta+\sqrt{(4+\delta)^{2}-16}}{2}
$$

A few comments are in order.

Comment 1 The competitive equilibrium satisfies the no restarting condition for all values of $\delta$, including $\delta=0$. This last case is of particular interest because it coincides with the monetary equilibrium allocation of the treeless economy, which as we saw did not satisfy the no restarting 
condition. The reason for this apparent contradiction lies in the role of a dividendless tree that only the old have. This is effectively the same as assuming that the first old can issue money and the later generations cannot. It is a very different environment from the treeless economy that we looked at first.

Comment 2 Both the competitive equilibrium and the organizational equilibrium allocations are Pareto optimal.

Comment 3 The organizational equilibrium yields much higher utility for the first generation than the competitive equilibrium, and the opposite holds true for the later generations. The difference between the two equilibrium concepts is that under our implementation of organizational equilibrium, there is no competition among the first old. Therefore, they capture all the gains from trade from later generations. The explicit introduction of competition among the first old in our environment would prompt the organizational equilibrium allocation to coincide with the competitive equilibrium. This point is closely related to the equilibrium concept used in Chari and Hopenhayn (1991).

\section{Non-Stationary Environments}

So far we have dealt only with economies that are stationary. The extension of the notion of organizational equilibria to these environments is not obvious: What does it mean that agents in the same situation as previous agents have to do as well as those agents? To partially address this issue, we provide in the next subsection an example of how a growing economy can be transformed into a stationary one to which the notion of organizational equilibria can be applied directly. After that we offer some thoughts on the extension of the notion to other types of nonstationarity. 


\subsection{Growing Economies}

We have established our result for stationary economies. An interesting extension of our equilibrium concept is to a class growing economies. Let an economy be growing by a constant factor $\gamma>1$. This means that the endowment of generation $t$ is $\gamma^{t}\left\{e^{y}, e^{z}\right\}$. Assume that preferences of generation $t$ over goods $\left(x_{t}, x_{t+1}\right)$ can be represented by a homogenous of degree one, strictly quasi-concave function $u\left(x_{t}, x_{t+1}\right)$.

We proceed to transform this growing economy into a stationary one. Let $\hat{x}_{t}=\gamma^{-t} x_{t}$ for all $t$. With this transformation endowments for all generations are $\left\{e^{y}, e^{z}\right\}$, which are stationary. Lemma: $\hat{u}\left(\hat{x}_{t}, \hat{x}_{t+1}\right)=u\left(\hat{x}_{t}, \gamma \hat{x}_{t+1}\right)$ represent preferences of generation $t$ over $\left(\hat{x}_{t}, \hat{x}_{t+1}\right)$.

Proof. The result follows from function $u$ being homogenous of degree one.

This establishes that the environment is stationary. The organization equilibrium is welldefined and is the solution to the program in section 6 .

\subsection{Other Types of Non-Stationarity}

Consider an economy like that in our main example except that the first agent has an endowment of $\{30,1\}$ rather than $\{3,1\}$. What allocation will our equilibrium pick? Note first, that $\log 30>2 \log 2$ which implies that no matter what, the first old will be better off than any agent after him, yet positive transfers from the young to the old are Pareto improving. The issue is, Can the first old obtain any transfers? The answer is yes. The first old could get as much as $\hat{x}_{1}=1 / 3$ (the first period transfer in our main example). The second old cannot do better by proposing the same plan.

What about the case when the endowment of the first old is $\{2,1\}$ ? What can the first old get? The first old could get one unit of the good as the transfer. Note that the second old could 
not get the same unit as a transfer because the second old has a bigger endowment than the first old. Note, moreover, that the first old does not attain higher utility than the second; if it did, the second old could destroy part of its endowment and restart the economy. The principle then that should follow of what it means to do as well in the same situation is that higher transfers can be attained as long as the utility is, not higher; otherwise, transfers cannot be larger. Note that in this example, things are relatively easy because both economies are identical from period 2 on. For general forms of non-stationarity it is much harder to implement the notion of doing as well if in the same situation.

\section{Conclusion}

In this paper we have argued that for stationary OLG type environments with endowments tilted towards the young, standard equilibrium concepts are not very good. We provide a new equilibrium concept, organizational equilibrium, based on voluntary participation in a coalition, suited for the OLG environment (and we hope others) that requires a form of participation constraints that is rooted in the notion that members have the option to start over. We have shown that for the standard OLG environments the equilibrium exists and is unique. We have also discussed how in the presence of assets, organizational equilibrium coincides with competitive equilibrium, a reassuring property. We also have some extensions to non-stationary environments. 


\section{Footnotes}

${ }^{1}$ On occasion we will make the interpretation of a measure one set of identical, atomical agents. This is not a crucial point since the key interactions are intertemporal. What is valid for one agent is valid for a measure one of agents, except in section 7 , where we show the equivalence of organizational and competitive equilibria.

${ }^{2}$ The literature has sometimes described this property as "printing their own money" (see Esteban (1986)).

${ }^{3}$ However, this notion has already been used in the literature by Becker (1995) with a very different meaning in economies with capital accumulation.

${ }^{4}$ Recall the subtitle of Samuelson's (1958) original piece.

${ }^{5}$ We use the Debreu (1954) definition of valuation equilibrium, which requires all markets to clear with equality.

${ }^{6}$ In fact we proceeded backward by finding the sequence of markets equilibrium and then implementing it as a valuation equilibrium by an appropriate choice of the commodity space.

${ }^{7}$ It may be interesting to point out where the proof of the first welfare theorem breaks down. It is at the point where the value of the commodities chosen by all agents is used, to show that it should be higher than that of the endowment which yields a contradiction. In this model economy the value of most feasible allocations, including both autarky and any Pareto superior one, is infinite, which breaks down the argument that yields a contradiction.

${ }^{8}$ Zakharova (2000) shows that in certain OLG environments with a state variable, the $T$ operator has a maximal fixed point. 


\section{References}

Asheim, G. 1997. "Individual and collective time-consistency," Review of Economic Studies, 64, $427-43$.

Becker, R. A. and Chakrabarti, S. K. 1995. “The recursive core,” Econometrica, 63 (2), 401-23.

Boldrin, M. and Rustichini, A. 2000. "Political equilibria with social security," Review of Economic Dynamics, 3, 41-78.

Chari, V. and Hopenhayn, H. 1991. "Vintage human capital, growth, and the diffusion of new technologies," Journal of Political Economy, 99 (6), 1142-1165.

Debreu, G. 1954, "Valuation equilibrium and Pareto optimum," Proceedings of the National Academy of Science, 40, 588-592.

Engineer, M., Esteban, J., and Sakovics, J. 1997. "Costly transfer institutions and the core in overlapping generations economies," Journal of Economics Behavior and Organization, 32 (2), 287-300.

Esteban, J. M. 1986. “A characterization of the core in overlapping generation economies: An exact consumption-loan model of interest with or without the social contrivance of money," Journal of Economic Theory, 39 (2), 439-456.

Hammond, P. 1975. “Charity, altruism or cooperative egoism?” in Phelps, E. S. (ed.), Altruism, Morality and Economic Theory. New York: Russell Sage Foundation.

Hendricks, K., Judd, K., and Kovenock, D. 1980. "A note on the core of overlapping generation models," Economics Letters, 6, 95-97.

Kocherlakota, N. R. 1996. "Reconsideration-proofness: A refinement for infinite horizon time inconsistency," Games and Economic Behavior, 15 (1), 33-54. 
Samuelson, P. 1958. "An exact consumption-loan model of interest with or without the social contrivance of money," Journal of Political Economy, 66, 467-482.

Shell, Karl. 1971. "Notes on the Economy of Infinity," Journal of Political Economy, 79, 10021011.

Silverman, D. 1999. “Note: Revision proofness and overlapping generations,” Mimeo, University of Pennsylvania.

Wallace, N. 1980. “The overlapping-generations model of fiat Money," in Kareken, J. H. and Wallace, N. (eds.), Models of Monetary Economies, 49-82. Federal Reserve Bank of Minneapolis, Minneapolis.

Zakharova, D. 2000. "Time consistency and up-or-out promotion," University of Minnesota and Federal Reserve Bank of Minneapolis working paper, May. 
Figure 1: Autarky (B) and stationary optimal (A) allocations.

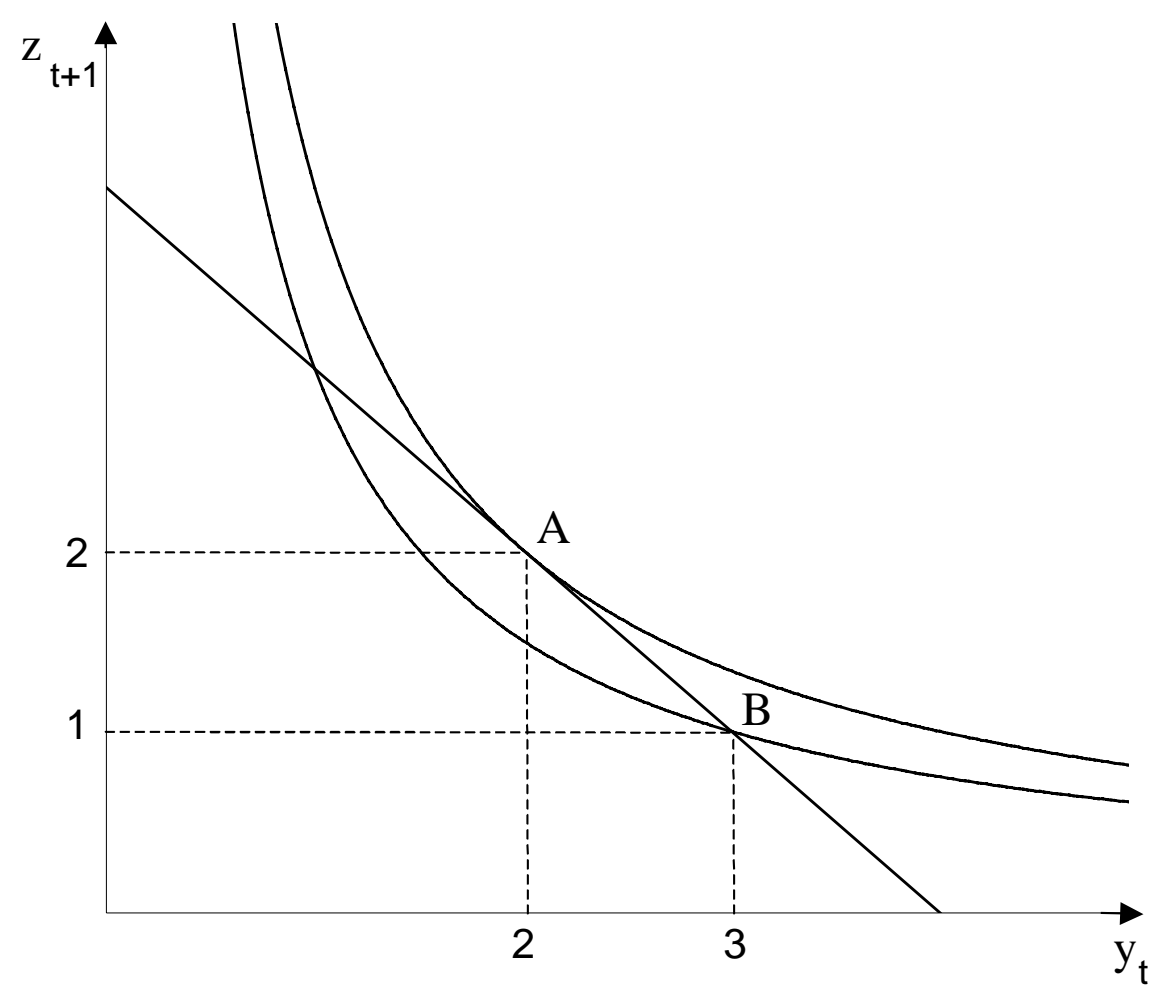


Figure 2: Proposed allocation.

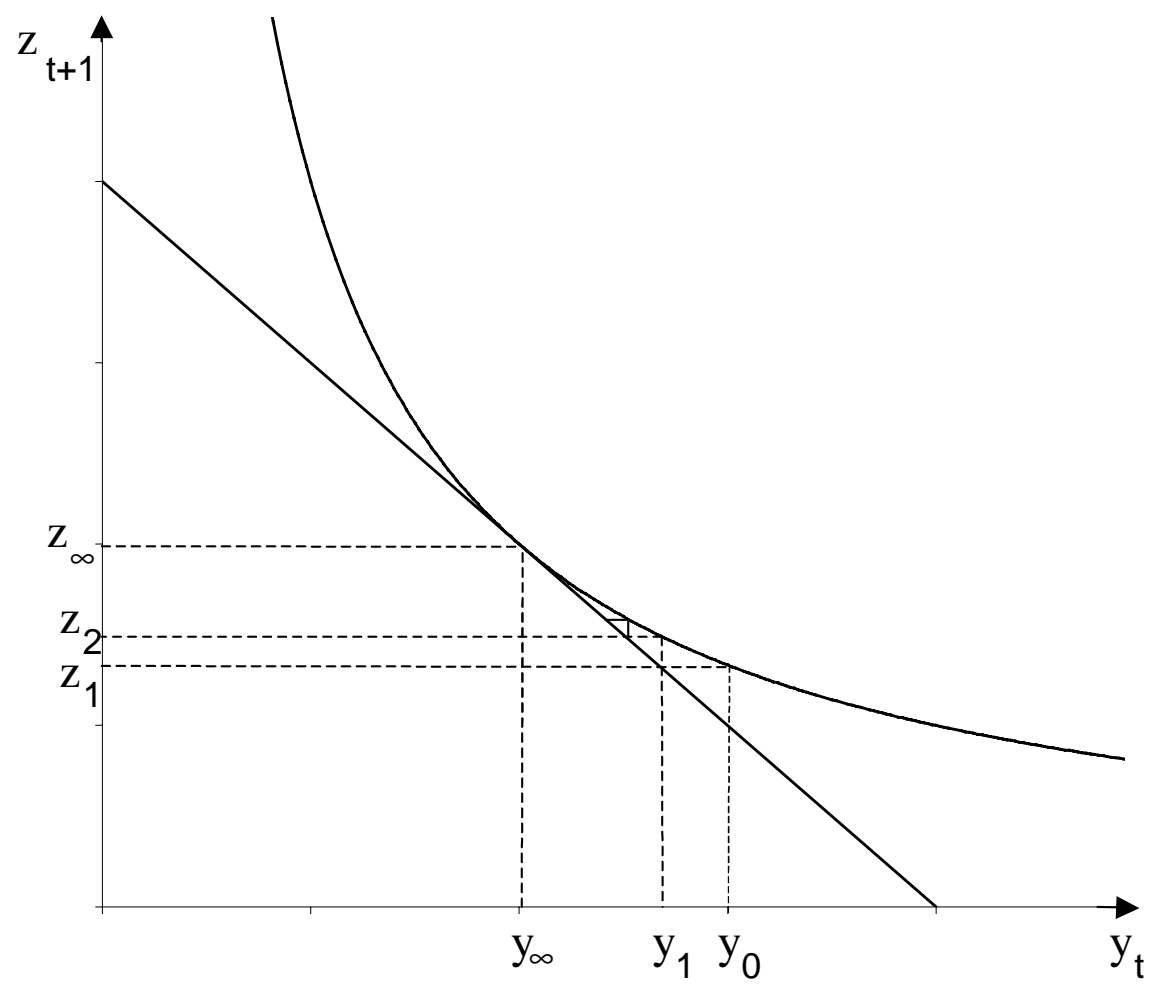

